\title{
APPLICATION OF GENERATIVE DESIGN FOR STRUCTURAL OPTIMIZATION AT THE CONCEPTUAL DESIGN PHASE
}

\author{
ANDERS SAGVÅG BIRKEMO ${ }^{1,2} \&$ SAMINDI M. K. SAMARAKOON ${ }^{1}$ \\ ${ }^{1}$ Department of Mechanical and Structural Engineering and Materials Science, University of Stavanger, Norway \\ ${ }^{2}$ Multiconsult Norge AS, Norway
}

\begin{abstract}
Optimization of structural components leads to higher performing designs, by reducing unnecessary cost and increasing the efficient use of material. The structural optimization process is a rigorous iterative time-consuming process. Developments within algorithmic aided design (AAD) tools have made it easier for structural engineers to achieve automation of optimization routines. Generative design (GD) provides optimization routines which can aid in the pursuit of optimal solutions, given a set of criteria. The use of GD in combination with finite element analysis (FEA), to optimize structural design, has yet to see noticeable adoption within the structural engineering community. This paper discusses two illustrative cases to demonstrate how GD tools have been applied to optimize structures at the conceptual phase. The results have been validated using FEM-design software. A workflow has been proposed, based on the lessons learnt from the cases. This workflow inherits characteristics that, when followed, yielded consistent and reliable results.
\end{abstract}

Keywords: algorithmic aided design, generative design, structural optimization, workflow.

\section{INTRODUCTION}

With the advent of new and more powerful technologies, designers and architects are empowered to realize increasingly complex structures [1]. Structural engineers are often tasked with taking such concepts from an idea to a product which is buildable and structurally sound, while adhering to the initial design. For structural engineers, the final delivery will often be in the form of a highly detailed and information-rich 3D model (model-based delivery), traditional 2D drawings, or a combination of the two. The purpose of such deliverables is to serve as the reference for the builders at the building site. Naturally, these references need to be of high quality and free from errors, while precisely mimicking the physical and functional characteristics of the building. To achieve this, all the aforementioned deliveries are regularly generated in some form of BIM software such as Tekla or Revit [2]. Such software offers easy collaboration between project team members and enables thorough quality control procedures. Consequently, to keep up in an environment where the bar is raised continuously, proficiency in such tools is fast becoming a necessity [3]. As a result, efficient modelling methodologies such as parametric design have an increasing presence in the typical structural engineering workflow. Furthermore, as this methodology is seeing more use, new and inventive workflows are proving to alter the ways structural engineers work on a daily basis. Moreover, structural engineers regularly use Finite Element Analysis (FEA) tools to evaluate the structural integrity of structures. To do so, analytical models must be generated. In much the same manner as BIM models, such analytical models can be made parametrically. In addition, by having an algorithmically defined structure, additional benefits are enabled. Since the position of all elements is governed by the logic described by the script, optimization algorithms can be deployed which in turn can manipulate the geometry to achieve certain objectives. Structural engineers aim to optimize structures in terms of the amount of 
material needed while maintaining the structural integrity of the building. To analyze and design structures, structural engineers commonly use FEA software. In other words, for the optimization algorithm to properly assess each iteration of the structure, FEM analyses need to be incorporated into the automated workflow. With the use of Grasshopper plug-in Karamba3D, such capabilities are enabled. With the power of Grasshopper, Karamba3D and optimization algorithms such as simulated annealing and evolutionary solver, structural engineers can let the algorithms turn out solutions in the conceptual design phase. Furthermore, being able to deploy algorithms which can aid in the finding of optimal solutions can provide significant yields, in terms of both the overall price of the structure and the time savings related to the conceptual design phase. Furthermore, having integrated structural analysis within the parametric modelling routine will enable high levels of interoperability between the Building Information Modelling (BIM) model and the analysis model.

Through case studies, this paper aims to illustrate workflows structural engineers might adopt in the future. Although the applications shown are limited, the fundamental purpose is to highlight the possibilities that exist in the employment of optimization algorithms in structural design. Finally, a general workflow will be suggested that describes how to attack a problem, with the aforementioned algorithms.

As this paper mainly conducts research through case studies, qualitative research will be the overarching method; this also falls in line with the use of surveys to obtain feedback from industry professionals on the different topics. However, to evaluate the accuracy of the case studies, quantitative methods are needed to compare the numerical results from various sources. In other words, the quantitative research method will be used to validate the outcome of certain cases, to be able to qualitatively evaluate the cases as a whole.

\section{GENERATIVE DESIGN VERSUS TRADITIONAL METHODS}

Traditionally, the optimizing of structures is done iteratively, with a more trial-and-error approach. Obviously, through previous projects, an experienced engineer will have found solutions to problems that might resemble the task at hand. Their previous experience will then serve as a foundation for how to proceed in the pursuit of solving the said task. This approach is tried and proven and, with the collective experience of the project team, will reliably produce adequate results. However, past solutions do not necessarily mean that it is the most optimal way of solving the particular problem. In addition, it is next to impossible to rule out biases based on past experiences. Consequently, the exploration of other and possibly superior alternatives might be neglected, on the sole base that a deemed preferred solution has already been used. Generative design refers to the process of deploying optimization algorithms that aid in the search for optimal solutions, given a set of inputs and constraints, as shown in Fig. 1. Then, the optimization algorithms explore the possible solution space through the iteration of generational genome populations. A common method used in generative design is evolutionary solvers. Various generative design tools have been developed, as discussed in the following section, and the general approach can be seen in Fig. 1.

To implement generative design approach, there are variety of software combinations can be used. Moreover, Rhinoceros: 3D-CAD software developed by McNeel is mostly used in the industry and has long been a favourite among designers. In later years, with the inclusion of visual programming capabilities provided by the imbedded application Grasshopper, Rhinoceros has seen noticeable adoption among architects and civil engineers who seek to enhance their efficiency in modelling BIM models. In addition, FEA software 


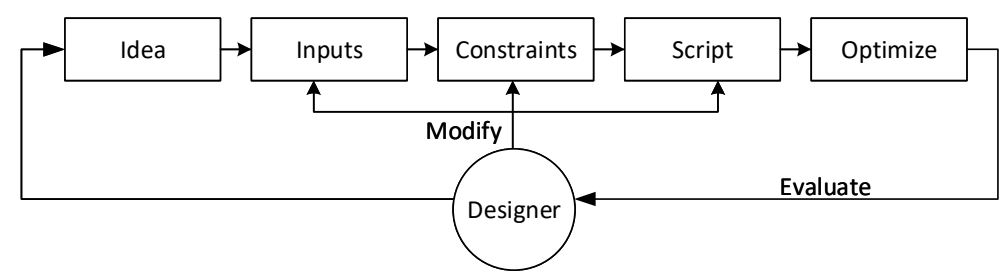

Figure 1: Generative design approach.

providers have also seen the potential and are now providing plug-ins that enable bidirectional interoperability between their respective software and the modelling capabilities that Rhinoceros brings. With the use of Grasshopper, the user can, with a high degree of precision and efficiency, develop scripts that produce complex geometry that would be cumbersome to do manually. It could be argued that Grasshopper is the go-to tool in terms of Algorithmic Aided Design (ADD) and has by far the largest community support, compared to its competitors. Furthermore, Galapagos is an optimization routine, also developed by Rutten [4], which is embedded within Grasshopper. Galapagos enables the user to define a set of input parameters which the algorithm can manipulate. Furthermore, these parameters are usually inputs to a problem which needs optimization. For Galapagos to learn which combination of the parameters yields the best solution, a fitness criterion needs to be defined. The fitness criterion discusses the performance of that particular iteration, given the associated parameter values. Subsequently, when the algorithm is set up as described, the routine can be set in motion. Before starting, Galapagos asks whether the fitness score should be minimized or maximized. Finally, the user can choose from two different optimization techniques, evolutionary solver or simulated annealing. Evolutionary solver is by far the most common method and is also the one applied to the relevant cases in this paper.

Karamba3D is an FEA plug-in for Grasshopper [5] which enables scripting of structural analysis in the same manner as is regularly done with geometry within Rhinoceros. It is very "lightweight", providing fast solutions to structural analysis problems defined by geometry and information programmed in a Grasshopper script. This capability makes it a prime candidate to be paired with generative design routines like Galapagos. Exploiting this capability will be further studied in this paper. This study focuses on the application of generative design, using Karamba3D, Galapagos and Grasshopper for the optimization of structural members.

\subsection{Collaboration of BIM models with digital tools at conceptual design phase}

Conceptual design is the starting point of the structural design process, at which design requirements given in the relevant standards (i.e., strength, durability, robustness, and safety) and the client's demands are taken into account. During the modelling process at the conceptual stage, it is vital to have a fruitful collaboration with the architect, structural engineer, and client, to discuss all the various considerations, to achieve the optimal design. However, it has been challenging to achieve productive collaboration during the conventional structural design process because of the lack of information/data exchange and poor visualization of the end product. In this case, the use of BIM-based structural design enhances the collaboration among different participants in a project. Moreover, currently, BIM-based structural design has been widely-adopted in construction practice, to 
overcome such challenges and to achieve customer satisfaction. Furthermore, the use of BIM during structural design results in systematic modelling processes, a powerful interactive visualization platform, and standardized data exchange interfaces during the life cycle of the construction. However, the benefits of using generative design for structural optimization and integrating of BIM have not been well understood because of the lack of knowledge about workflows and the use of different digital tools. Particularly during the conceptual design phase, as given in Fig. 2, digital tools can be used to implement a generative design approach to achieve optimized structural solutions while collaborating with BIM models.

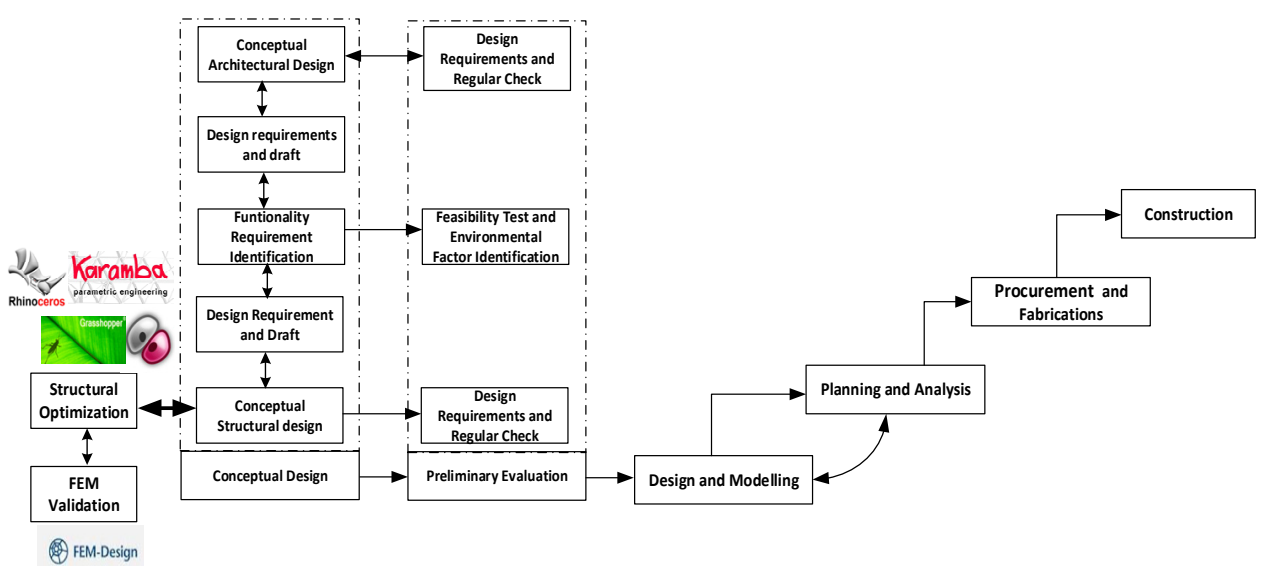

Figure 2: BIM-enabled structural design in construction. (Source: Modified from Chi et al. [6]).

\section{ILLUSTRATIVE CASES TO IMPLEMENT GENERATIVE DESIGN FOR STRUCTURAL OPTIMIZATION}

To study the potential of structural optimization algorithms, a set of cases has been constructed. These cases were developed with input and guidance from industry professionals and stem from real cases they have experienced in their work. To study these cases, a flowchart has been established, as given in Fig. 3. According to Fig. 3, case description gives a brief description of the problem at hand which includes an explanation of the structure and why the problem is suitable for optimization. Then, to being the optimization, it is necessary to idealize the case and set limitations. Commonly, structural engineers idealize the structure when performing the structural analysis; the case-specific idealizations that have been made, in addition to the limitations of the procedure, will be described during this stage. During this process, it is vital to set the optimization goal, based on the target outcome.

For each optimization problem, the algorithm needs a set of inputs at the beginning. Furthermore, these inputs are classified as either static or variable. Static inputs are parameters that remain unaltered through the optimization process, effectively functioning

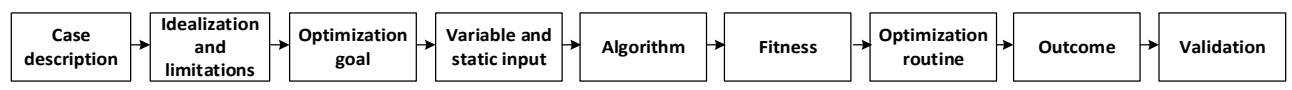

Figure 3: Flowchart for optimization of structural members. 
as the constraints to the given problem. Variable inputs, however, are the parameters the optimization algorithm can alter in the pursuit of the most optimal solution. Whether the inputs are static or variable will depend on the optimization goal and are therefore casespecific. Moreover, algorithms can be used to produce an array of design options based on the requirements set at the beginning. The performance of the structure will be a sum of parameters deemed important for the value of the given iteration. Parameters such as passing the preliminary Serviceability Limit State (SLS) check, as well as the total weight of the structures, are examples of ways to measure the performance of the iteration. After the algorithm has run its course, the best solution, with its accompanying genes and fitness, is presented. To validate the result, stand-alone FEA software such as FEM-Design is used. The top solution proposed by the optimization algorithm will be exported through automated workflows enabled in Grasshopper, and more thorough analyses can be undertaken.

\section{CASE 1: STRUCTURAL OPTIMIZATION OF 65-M STEEL TRUSS WITH RESPECT TO SELF-WEIGHT}

The steel truss shown in Fig. 4(a) is $64.6 \mathrm{~m}$ in length, $4.8 \mathrm{~m}$ high, and there is $9.6 \mathrm{~m}$ distance between adjacent truss members. It has been targeted to optimize the weight of the steel truss using a generative design approach, as discussed in Fig. 3, and using the software given in Section 2.5. In this case, the goal of the optimization was to find the lightest steel truss by satisfying the constraint of $100 \mathrm{~mm}$ maximum displacement due to the applied loads. The load used in this analysis is weight of the roof of $1 \mathrm{kN} / \mathrm{m}^{2}$ and snow load of $2.8 \mathrm{kN} / \mathrm{m}^{2}$. Moreover, the yield strength of steel in the truss is $355 \mathrm{MPa}$ (S355). During this analysis, one load combination was chosen. However, the analysis can be carried out for all the load combinations given in most of the standards, to obtain an optimized solution.

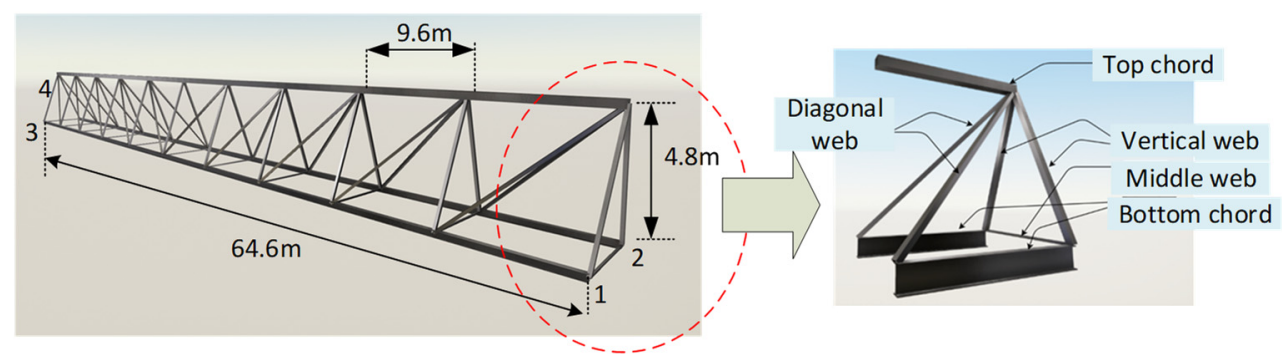

(a)

(b)

Figure 4: (a) 3D view of the steel truss; and (b) Different chord in the truss.

Initially, length, height, distance between truss members and loads were assigned as static input to Grasshopper software. Although these static inputs remain static throughout the optimization, they could easily be changed to suit a new and different sized truss with alternative imposed loads. The steel truss was allowed to rotate major axis and supported over pin and roller supports. The steel truss was subjected to loading from both the roof and the snow that could accumulate on it.

It is vital to define variables at the beginning of the analysis in Grasshopper software. In this analysis, input variables, such as number of bays in truss (10-20), height $(4.8 \mathrm{~m}-$ $6.64 \mathrm{~m}$ ) of the truss, and cross-truss members at top, bottom, middle, vertical and diagonal 
have been used. The algorithm got two genes to alter, in order to find the most optimal solution: the total number of truss bays and the height of the truss.

To optimize the structure, the algorithm could use a pool of different cross sections (see Fig. 4(b)), the diagonal web members, vertical web members, and middle web members, and the top chord could pick from a list of rectangular hollow sections (RHS), while the bottom chord could pick from a pool of HEB sections. The different structural members, as well as the span of possible cross sections have been given in Table 1.

Table 1: Variable input: cross sections.

\begin{tabular}{|l|l|}
\hline Member & Span of values \\
\hline Diagonal web & {$[$ RHS20 $\times 20 \times 2$, RHS400 $\times 400 \times 16]$} \\
\hline Vertical web & {$[$ RHS20 $\times 20 \times 2$, RHS400 $\times 400 \times 16]$} \\
\hline Middle web & {$[$ RHS20 $\times 20 \times 2$, RHS400 $\times 400 \times 16]$} \\
\hline Top chord & {$[$ RHS20 $\times 20 \times 2$, RHS400 $\times 400 \times 16]$} \\
\hline Bottom chord & {$[$ HEB100, HEB1000 $]$} \\
\hline
\end{tabular}

\subsection{Algorithms for optimization of the weight of the steel truss}

Based on the input variables summarized previously, a generative algorithm was constructed for optimization of the steel truss via the technical route of the random generation of parameters within the given range and selection by constrained conditions. The algorithm is based on Grasshopper to realize the automatic generation of different steel trusses based on the input variables. The framework of the algorithm is shown in Fig. 5.

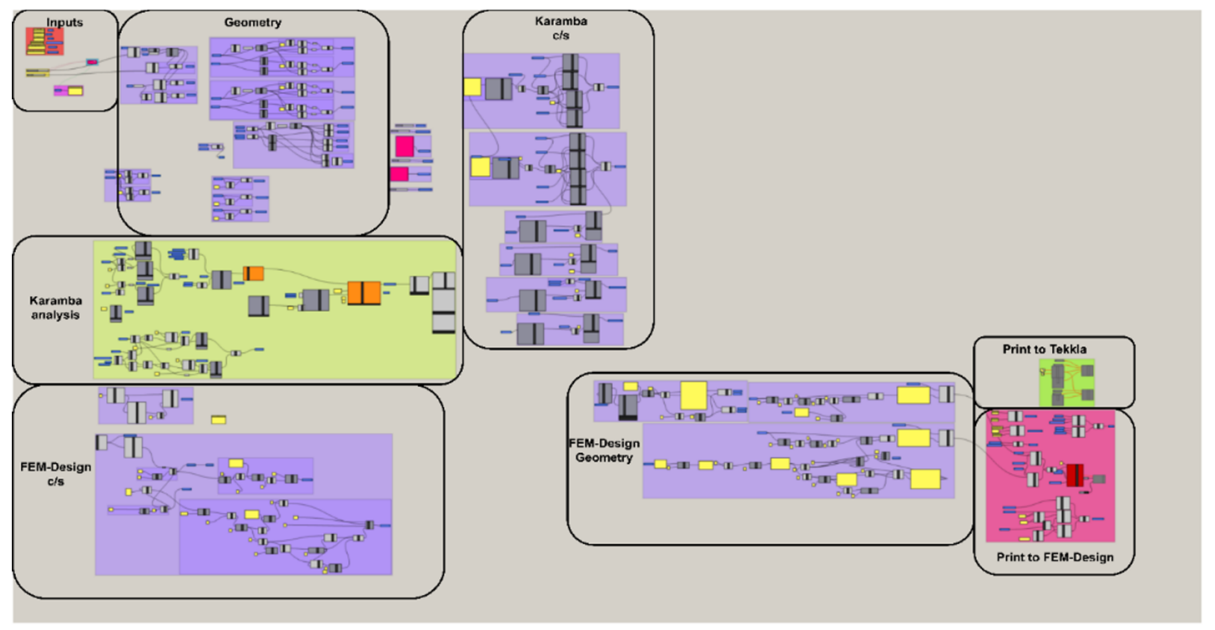

Figure 5: Case 1-Framework of the algorithm constructed to optimize the weight of the steel truss.

According to Fig. 5, first the lines which will later represent truss members are drawn up, and all the top points where the diagonals intersect the top chord are gathered. Furthermore, all lines are then assigned a random cross section picked from a pool of predefined sections. Subsequently, self-weight and snow load are applied as point loads to 
the previously gathered top points of the truss. Depending on the number of truss bays, the magnitude of the loads will vary. The location of the support points is set, and supports are made with the appropriate degrees of freedom. Now Karamba3D has enough information to evaluate the structure. When it is finished, Karamba3D determines the best combination of cross section for each member to meet the constraint of maximum displacement while not exceeding the utilization of each member. When all members have been given a new cross section, the total mass of the structure is calculated. The total mass will be the fitness of that particular iteration and is sent back to Galapagos. This is repeated until it finds the lightest structure that still fulfils the requirements. Once Galapagos has found a suitable solution, it is sent to FEM-Design for validation. In addition, if needed, the structure can also be sent to Tekla to populate a BIM model.

\subsection{Optimization routine using Galapagos}

The optimization routine makes use of the evolutionary solver within Galapagos. The objective of this analysis was to obtain the lightest structure by satisfying the given constraints, i.e., the algorithm was set to minimize the fitness value. The total population of each generation was bound by a maximum of 50 genomes; this should be sufficient to properly explore the fitness landscape, while keeping the runtime of the optimization routine within reasonable limits. The initial boost was set to 4 ; i.e., generation 0 will have four times as many genomes as the following generations. This was done to thoroughly cover the fitness landscape such that the following generations did not colonize local optima instead of the best global one. With a total runtime of 15 minutes, the evolutionary solver converged towards a solution after 41 generations, yielding a total weight of $77516.56 \mathrm{~kg}$. After running the optimization routine, the fittest genome was found to be when height was $6.64 \mathrm{~m}$ and number of bays in truss was 10 .

\subsection{Optimized cross sections for steel truss}

The fittest genome and the steel truss including the applied load were found. Then, Karamba3D assigned the following cross sections to the different structural members in the steel truss, as shown in Fig. 6. The optimized cross sections obtained for different truss members are shown in Table 2 for half of the truss members; member ID is given based on Fig. 6.

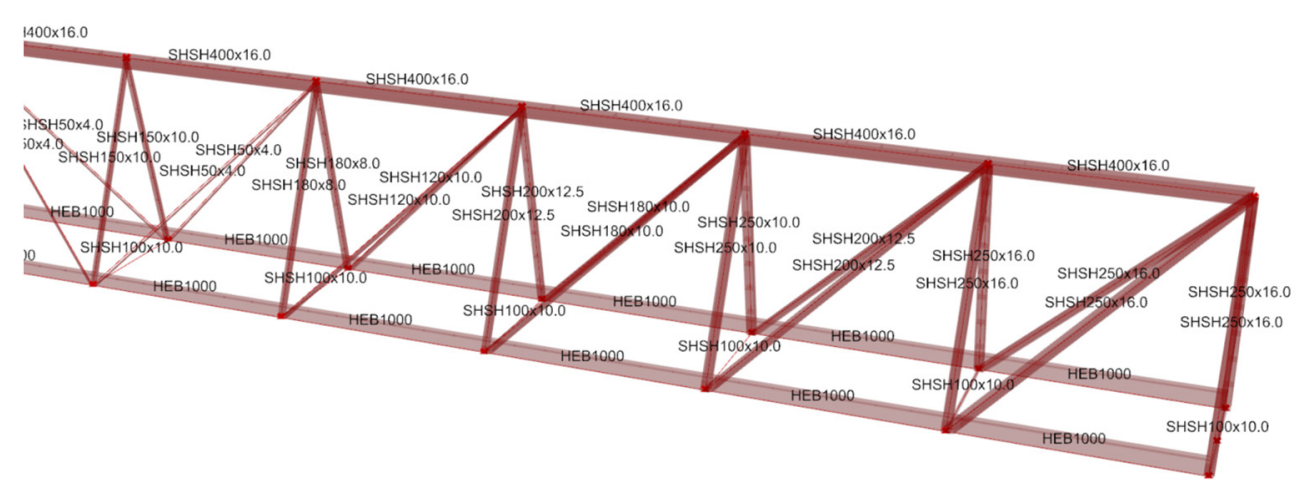

Figure 6: Case 1 - Optimized cross sections in half of the truss. 
Table 2: Case 1 - Optimized truss members in half of the truss.

\begin{tabular}{|c|c|}
\hline Member ID & Profile \\
\hline Diagonal 1 & SHS250 $\times 250 \times 16$ \\
\hline Diagonal 2 & SHS200 $\times 200 \times 12.5$ \\
\hline Diagonal 3,4 & SHS180 $\times 180 \times 10$ \\
\hline Diagonal 5 & SHS50 $\times 50 \times 4$ \\
\hline Vertical 1,2 & SHS250 $\times 250 \times 16$ \\
\hline Vertical 3 & SHS250 $\times 250 \times 10$ \\
\hline Vertical 4 & SHS200 $\times 200 \times 12.5$ \\
\hline Vertical 5 & SHS180 $\times 180 \times 8$ \\
\hline Middle & $100 \times 100 \times 10$ \\
\hline Top chord & $400 \times 400 \times 16$ \\
\hline Bottom chords & HEB1000 \\
\hline
\end{tabular}

4.3 Validation of the results using FEM-design software

The optimized steel truss was further validated using FEM-Design software. To validate the results, the geometry, loads, cross sections and supports were exported to FEM-Design software. The process was automated through the FEM-Design plug-in for Grasshopper. The results from the SLS load combination yielded a maximum vertical displacement of 98 $\mathrm{mm}$. The results from the ultimate load combination yielded a maximum utilization of $68 \%$ (see Fig. 7). After the structural integrity was validated within FEM-Design, the truss was printed to Tekla.

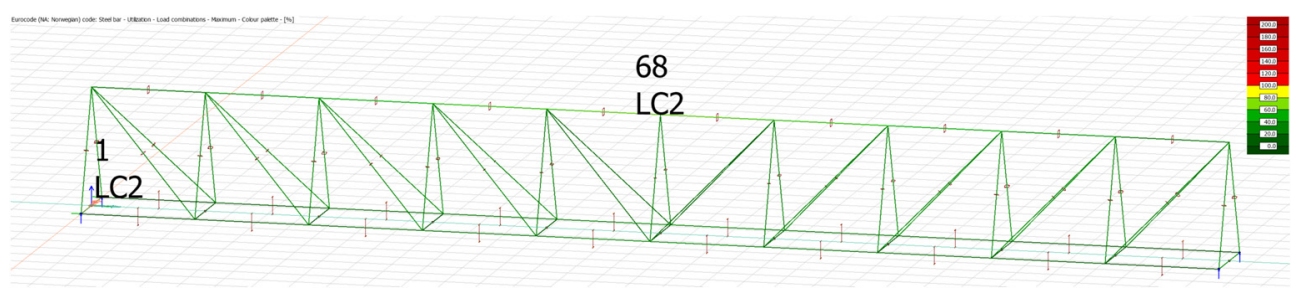

Figure 7: Case 1 - Validated result - Utilization.

\section{CASE 2: OPTIMIZATION OF TENDON PROFILE FOR A \\ THREE-SPAN POST-TENSIONED BEAM}

To accommodate large open areas within buildings, columns need to be placed further apart to increase the span length. Consequently, long-spanning beams are needed. Using reinforced concrete beams for long span lengths is not an economical solution. Consequently, structural engineers often turn to post-tensioned systems to solve this problem. These systems offer the engineer options to achieve structural integrity while keeping the beams relatively slender. The process of designing post-tensioned beams is performed iteratively, with the main outcome being to determine the necessary crosssectional area of the beam, the tendon profile, and, finally, the required tendon force. Initially, some assumptions regarding losses, trial cross section and tendon profile are made. Furthermore, the necessary tendon force is calculated to balance the loads vs. the applied forces. Finally, as the geometry of the tendon and the required force are known, the 
actual losses can be calculated. Compared to the initial assumptions, the engineer can now go back and re-iterate, to optimize the structure further. This process continues until the performance of the solution is deemed acceptable. The following case will present an automated approach to designing a post-tensioned system. The case draws inspiration from a real structure.

\subsection{Optimization of tendon profile in pre-stressed concrete beam}

A three-span pre-stressed concrete beam (i.e., post-tensioned) with parabolic tendon profile has been studied, as shown in Fig. 8. In reality, the distributed load acting on the beam by the post-tensioned tendon is not uniform, i.e., it varies with the slope. In other words, the idealization that has been carried out in this case is that the distributed loads are uniform within each parabola section. Furthermore, the optimization routine does not include losses in tendon force. In addition, the beam is only subjected to one load combination (SLS) during the optimization. For this case, the optimization goal was to find a tendon profile that needed the least amount of jacking force while still maintaining compression throughout the beam.

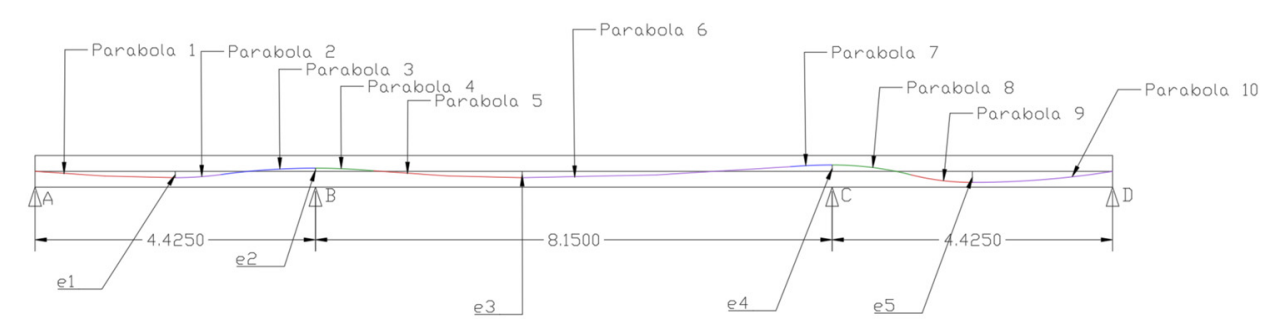

Figure 8: Case 2 - Post-tensioned beam (dimensions in meters).

In this study, span lengths of the post-tensioned beams have been selected as $4.42 \mathrm{~m}$ and $8.15 \mathrm{~m}$, as shown in Fig. 8. The post-tensioned beam is supported over four columns (i.e., pinned support), and the parabolic tendon profile consists of ten different parabolas. Moreover, a T-shape beam cross section has been used in this study, as shown in Fig. 9.

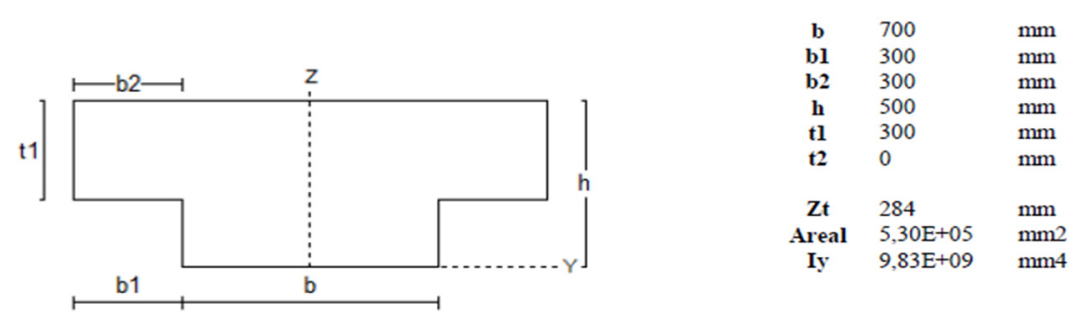

Figure 9: Case 2 - Static inputs - Cross section of beam.

In addition to the self-weight of the beam, three concentrated loads were placed $3 \mathrm{~m}$ from "A" with a magnitude of $210 \mathrm{kN}$ in " $A B$ " span, at $8 \mathrm{~m}$ from "A" with a magnitude of $115 \mathrm{kN}$ in "BC" span, and at $14.095 \mathrm{~m}$ from " $\mathrm{A}$ " with a magnitude of $115 \mathrm{kN}$ in "BC" 
span. The characteristic compressive strength of concrete has been used as $35 \mathrm{MPa}$. When designing pre-stressed concrete structures, it is vital to control stresses at SLS. In this case, the post-tensioned beam has been designed in such a way to maintain zero tensile stresses across the cross section of the beam. During the analysis, the partial factors of safety for permanent load: 1 and variable load: 1 were used in the load combination in the optimization routine.

To be able to optimize the tendon profile, the algorithm can alter a set of variables. To define the shape of the tendon profile, the algorithm can alter the location of a set of points (i.e., e0, e1, e2, etc.) of interest. The variable inputs for the algorithm were set up in such a way that they could alter the locations of the given points of interest by a fraction of the dimensions of the given structure, e.g., span length and cross-sectional height. Doing so meant that it would be a simple task to optimize a different beam by altering the span length and cross section.

\subsection{Algorithm for optimization of tendon profile}

To optimize the tendon profile using the algorithm shown in Fig. 10, the practical tendon profile described in Gilbert [7] has been used. Gilbert [7] describes how it is possible to idealize the tendon profile as parabolas with a given radius of curvature. Subsequently, the radius of curvature, together with the applied tendon force is directly related to the equivalent uniformly distributed load acting on the beam from the tendon. The algorithm uses the equations making up the approach and makes it available to the optimization routine. Initially, after all variable and static inputs have been set, the algorithm is set in motion.

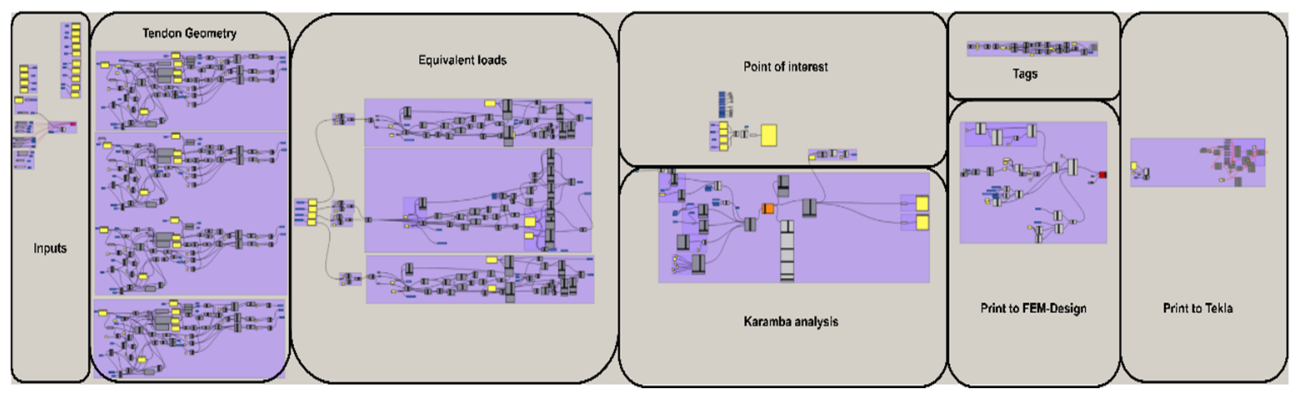

Figure 10: Case 2 - A framework algorithm.

\subsubsection{Procedure for constructing algorithm}

Gilbert [7] discussed a method to find a practical cable profile when pre-stressed concrete beams consist of different parabolic segments, as shown in Fig. 11. This method was used while developing the algorithm. In this method, initially, the distance $\left(h_{i_{1}}\right)$ which inflection point " $\mathrm{i}_{\mathrm{i}}$ " is offset " $\mathrm{e}_{2}$ " is calculated using eqn (1). Then, the algorithm has all the variables it needs to calculate the radius of curvature for the three parabolas, $r_{1}$ (parabola 1$), r_{2}$ (parabola 2$)$ and $r_{3}$ (parabola 3 ) in span $\mathrm{AB}$, using eqns (2)-(4) respectively. 


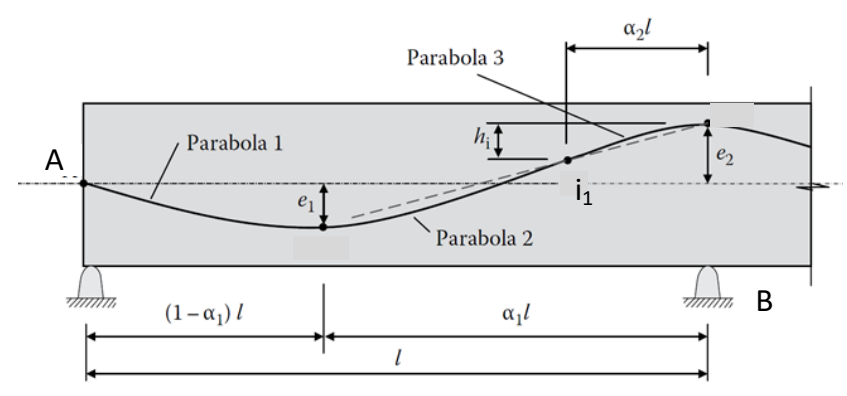

Figure 11: Case 2 - Illustration of tendon profile parabolas [7].

$$
\begin{gathered}
h_{i_{1}}=\frac{\alpha_{2}}{\alpha_{1}}\left(e_{1}+e_{2}\right) . \\
r_{1}=\frac{l^{2}\left(1-\alpha_{1}\right)^{2}}{2 e_{1}} . \\
r_{2}=\frac{l^{2}\left(\alpha_{1}-\alpha_{2}\right)^{2}}{2\left(e_{1}+e_{2}-h_{i_{1}}\right)} . \\
r_{3}=\frac{\alpha_{1} \alpha_{2} l^{2}}{2\left(e_{1}+e_{2}\right)} .
\end{gathered}
$$

When the radius of curvature is known, the equivalent uniformly distributed loads can be calculated using eqn (5). Similar equations have been developed for Span "BC" and "CD", and the algorithm has been constructed.

$$
q_{i}=P k_{P_{i}}
$$

where $P=$ Effective pressing force in post - tensioned tendon and $k_{P_{i}}=\frac{1}{r_{i}}, \mathrm{i}=1,2,3$.

Using the coordinates of the eccentricities and inflection points, together with the now known radius of curvature for each parabola, all parabolas can be drawn as curves in Rhino software. These curves will later be used to print the tendon shape to Tekla software.

\subsubsection{Use of Karamba3D}

Once the coordinates for each parabola and equivalent loads are calculated, it is necessary to develop the FEA model. Individual beam elements are modelled between each of the parabolas, ten in total. Furthermore, the equivalent loads and point loads are applied. The outcome of the analysis is the stress state at predefined points of interest. These stress states are finally used to evaluate the performance of the structure and enable Galapagos to find the most optimal solutions. Furthermore, the equivalent uniformly distributed loads are now made available to the FEA nodes in the Karamba3D plug-in. Together with the predefined beam elements and boundary conditions, the Karamba3D structural analysis node evaluates stresses in the cross section at 12 points of interest. The stress conditions, together with the applied tendon force, are fed to Galapagos as the fitness for the optimization.

\subsection{Fitness}

To measure the performance of each iteration, a fitness condition is constructed. In this case, the fitness conditions depend on two different inputs. The first input is the effective 
pre-stressing force in the post-tensioned tendon, the second is a sum of a condition-based equation determined by the stress condition at the 12 points of interest. For every point with stress above $0 \mathrm{MPa}$, i.e., tensile stress, a penalty value of 2,000 is added, e.g., if all 12 points are in tension, a penalty of 24,000 will be added. On the other hand, if all points are in compression, no penalty is added.

\subsection{Optimization routine}

When the variable inputs have been set up and a way to assess the performance of each iteration has been added, Galapagos has enough information to start the optimization routine. Galapagos has control of all the variable inputs and is given instant feedback on the performance through the fitness condition. Consequently, it gradually learns which combination of the variables yields the best performance and, after a set amount of time, presents the best solutions it could find. After a runtime of 45 minutes and 103 generations, Galapagos converged towards a solution only requiring $1,007 \mathrm{kN}$ of force. The magnitude and position of each equivalent load are presented in Fig. 12.

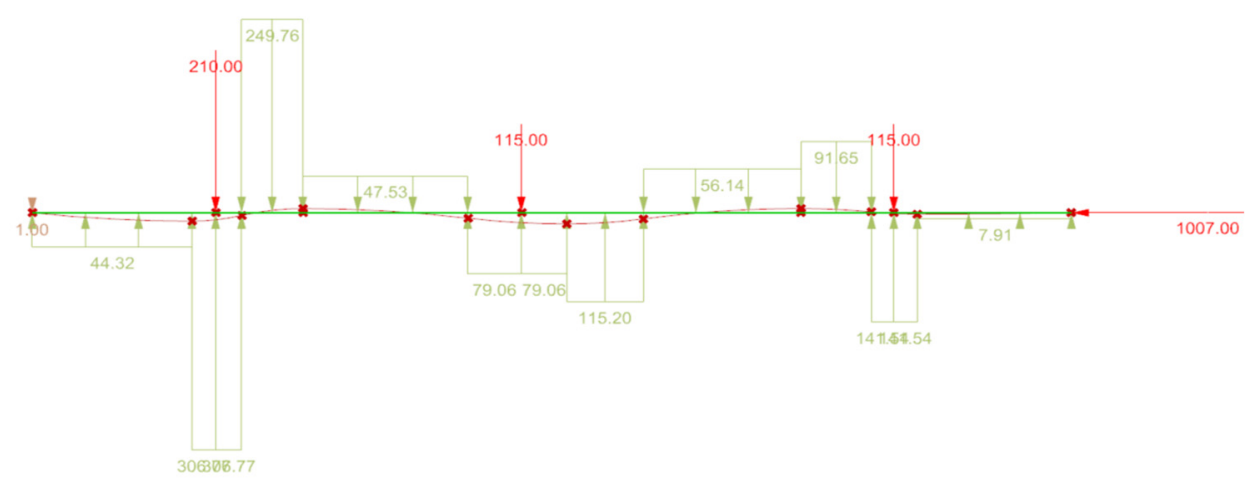

Figure 12: Case 2 - Illustration of loads ("red" color numbers are given in "kN", and "green" color numbers are given "kN/m").

\subsection{Validation of the optimized tendon profile}

To validate the result, the beam was also analyzed in FEM-Design software. The geometry of the structure, loads and load cases were sent automatically through the FEM-Design Grasshopper plug-in. However, it is not currently possible to model post-tensioned cables through the plug-in. The tendon profile and equivalent forces were therefore manually generated inside FEM-Design with the known locations for the points of interest. After running the analysis, the results given in Fig. 13 were found. According to Fig. 13, not only is the entirety of the beam in compression as intended, the minimum tensile stress is $0 \mathrm{MPa}$, indicating a well-optimized profile and tendon force. Finally, the geometry was automatically printed to Tekla to populate a BIM model.

\section{DISCUSSION}

In the pursuit of exploring the capabilities of Algorithmic Aided Design, this paper has presented two cases in which the generative design approach has been used for the 


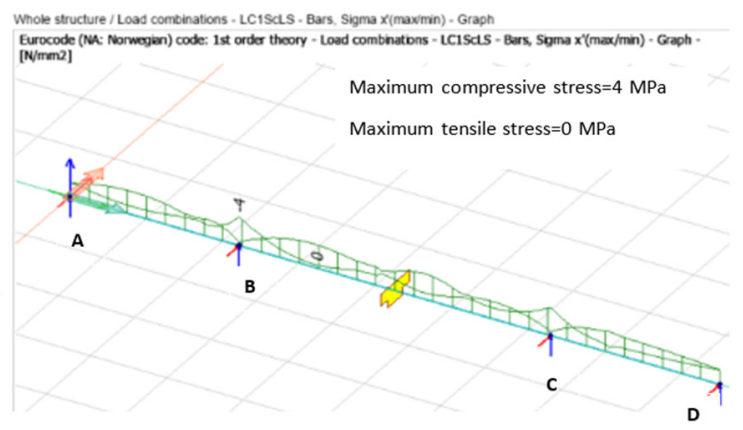

Figure 13: Case 2 - Validation of results.

optimization of structural members. A video demonstrating cases 1 and 2 can be seen at https://youtu.be/wIo5PulGBrM. After developing the scripts and algorithms and obtaining feedback from industrial professionals, the potential use of each case study in practice is discussed below.

\subsection{Case 1 - Structural optimization of $65-\mathrm{m}$ steel truss with respect to self-weight}

The process of setting up this script provided great insight into the intricacies of using optimization routines. The script was not only set up to optimize the structural members, but it also had to find an efficient way to gather the optimized structure and export it to FEM-Design for validation. Lastly, by using Karamba3D to select the optimized cross sections, the number of free variables, i.e., choosing the profile of each structural member, was greatly reduced. Consequently, the runtime of the optimization routine was significantly accelerated.

\subsection{Case 2 - Optimization of tendon profile for a multi-span post-tensioned beam}

Developing the script to run the optimization algorithm was quite time-consuming; in fact, the traditional way would probably be faster. However, since the script is set up in such a way that it is easy to change the loadings, dimensions, and span lengths, in future there is a possibility to use the developed script with some modifications for a similar optimization. As a result, significantly less time is needed to set it up. Furthermore, the results show that there is a satisfying correlation between the stress evaluation in Karamba3D and that in FEM-Design.

\subsection{Feedback and observations}

A questionnaire was sent out to obtain feedback on structural engineers' (i.e., Multiconsult, Norway) point of view about the use of optimization routines. Most of the structural engineers gave positive feedback about the cases studies and considered Generative Design a good tool in the conceptual design phase or in cases where a reduction in material costs is a significant priority. However, some hurdles still stand in the way of using such algorithms, such as the complexity of the scripts and the time needed to define them. However, presently, the structural engineers lack knowledge and training on developing 
optimization routines. Therefore, proper training will be able to overcome such hurdles in the future.

Both cases 1 and 2 show that it is indeed possible to set up routines to find reasonably good solutions to a problem, given a set of variables and constraints. However, the discussion of these cases was not necessarily about solving the presented problems but on illustrating the degree of automation a structural engineer can enable with the technology currently available. With the ever-growing prevalence of Machine Learning and Artificial Intelligence, automation has and will affect all industries; it is therefore naïve to think that structural engineering will somehow escape this. Consequently, to remain competitive, similar workflows should be explored to harvest the benefits, once the technology has reached adequate maturity. The cases described here can be considered stepping stones to fully automized machine learning algorithms solving structural engineering problems autonomously.

\subsection{Proposed workflow based on case 1 and case 2}

A workflow has been proposed based on two case studies, as shown in Fig. 14. Initially, static inputs are defined based on the type of optimization problem. Then, variable inputs (genes) and the range of their values should be defined. The inputs define the structural problem which is fed into Karamba3D. Moreover, Karamba3D analyzes the structure and outputs performance parameters in real time. Together with the performance parameters, some of the variable inputs (genes) will often make up the fitness of a given genome. The fitness and variable inputs (genes) are made available to the optimization routine, Galapagos. The optimization routine is run with a given optimization goal, either maximize or minimize. After the optimization routine has finished, the top solution is sent to FEMDesign for validation. After the structure has been validated, calculation reports can easily be generated.

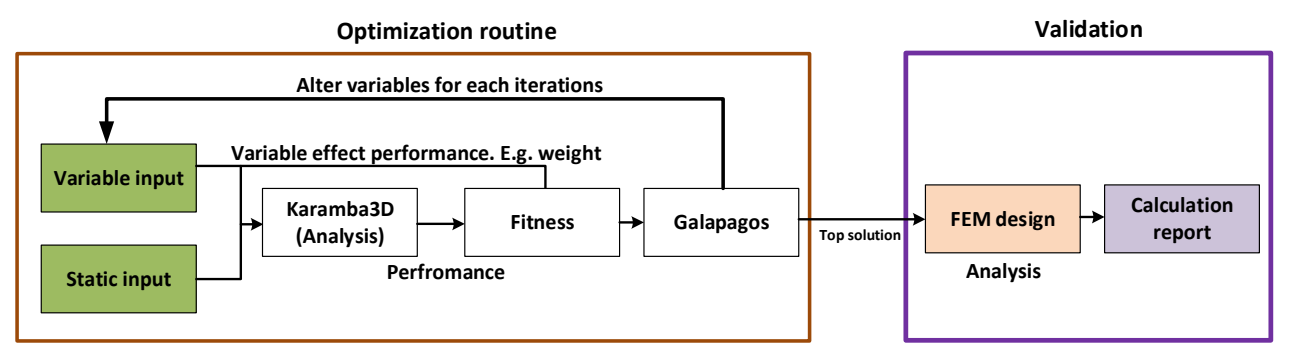

Figure 14: Proposed workflow for optimization of structural members using generative design approach.

\section{CONCLUSION}

The objective of this paper has been to explore cases, to obtain an understanding of how $\mathrm{AAD}$ is currently used, which possibilities it brings, and, lastly, to investigate what the future might hold. By having the analysis model defined parametrically, new and innovative technologies are enabled. Generative design, which is one of these, was explored in cases 1 and 2. Furthermore, these cases were used to verify whether structural optimization routines could produce reasonable solutions. As the verified results indicate, both cases highlight structures that satisfy the given criteria. Machine learning and artificial 
intelligence will affect all industries in the future; the cases described in this paper give a taste of how structural engineers might deploy computers to tackle the problems of tomorrow. Lastly, as an outcome of this paper, a proposed workflow has been presented. The workflow has been developed through trial and error, when working on the different cases, and inherited characteristics that, when followed, yielded consistent results. Consequently, this workflow can serve as a user guide for newcomers looking to make use of the technologies studied in this paper.

\section{REFERENCES}

[1] Banfi, F., Fai, S. \& Brumana, R., BIM automation: Advanced modeling generative process for complex structures. 26th International CIPA Symposium on Digital Workflows for Heritage Conservation, Copernicus GmbH, pp. 9-16, 2017.

[2] Kovacic, I. \& Filzmoser, M., Designing and evaluation procedures for interdisciplinary BIM use - An explorative study. Engineering Project Organization Conference, Devil's Thumb Ranch: Colorado, pp. 29-31, 2014.

[3] Russell, D., Cho, Y.K. \& Cylwik, E., Learning opportunities and career implications of experience with BIM/VDC. Practice Periodical on Structural Design and Construction, 19(1), pp. 111-121, 2014.

[4] Rutten, D., Galapagos: On the logic and limitations of generic solvers. Archit. Des., 83(2), pp. 132-135, 2013.

[5] Preisinger, C., Linking structure and parametric geometry. Architectural Design, 83(2), pp. 110-113, 2013.

[6] Chi, H.L., Wang, X. \& Jiao, Y., BIM-enabled structural design: Impacts and future developments in structural modelling, analysis and optimisation processes. Archives of Computational Methods in Engineering, 22, pp. 135-151, 2015.

[7] Gilbert, I.R., Design of Prestressed Concrete to Eurocode 2, 2nd ed., CRC Press, 2017. 